\title{
Essais
}

\section{Récits biographiques et mémoire collective au sein d'une jeunesse libanaise}

Bruno Lefort

\section{(2) OpenEdition}

1 Journals

Édition électronique

URL : https://journals.openedition.org/essais/9198

DOI : $10.4000 /$ essais. 9198

ISSN : 2276-0970

Éditeur

École doctorale Montaigne Humanités

\section{Édition imprimée}

Date de publication : 15 mai 2013

Pagination : $33-51$

ISBN : 978-2-9544269-0-7

ISSN : 2417-4211

\section{Référence électronique}

Bruno Lefort, «Récits biographiques et mémoire collective au sein d'une jeunesse libanaise », Essais [En ligne], 3 | 2013, mis en ligne le 01 septembre 2021, consulté le 08 septembre 2021. URL : http:// journals.openedition.org/essais/9198; DOI : https://doi.org/10.4000/essais.9198 


\section{Récits biographiques et mémoire collective au sein d'une jeunesse libanaise}

\section{Bruno Lefort}

"L'état de l'attachement pour autrui ne s'explique pas " affirmait Gérard Lenclud dans un article portant sur les clans politiques en Corse ${ }^{1}$. Le lien, poursuivait l'auteur, ne peut se comprendre que dans le cadre de la construction d'une relation sociale, manifestée concrètement, entre deux individus. Plutôt que de chercher à décrire le lien social à travers sa dénomination - ici la nation, là le clan ou la famille - je me proposerai en conséquence, suivant une recommandation de Bogumil Jewsiewicki ${ }^{2}$, de me tourner vers ses processus de représentation, et ce afin de réinterroger les catégories d'analyse mobilisées pour rendre compte de l'attachement. La représentation peut ici s'entendre à la fois comme mise en scène et comme « la capacité de prendre avec légitimité la place de l'absent, [...] parce que sa nature collective fait qu'il ne peut être donné à voir que symboliquement (nation, prolétariat, etc.) $»^{3}$, qui implique une définition de la signification accordée à cette collectivité.

La mise en récit du lien, entendu comme le résultat d'un processus d'intégration matériel et symbolique de l'individu au sein d'un collectif, constitue un des modes de représentation privilégiés de l'attachement au groupe. Non seulement permet-elle de " dire » son sentiment d'appartenance, mais elle manifeste également l'inscription de l'individu au sein de réseaux de sociabilités constitutifs de l'affiliation, la nature de cette double insertion ${ }^{4}$ définissant le

1 Gérard Lenclud, "S'attacher. Le régime traditionnel de la protection en Corse ", Terrain [en ligne], 21, 1993, p. $12:$ http://terrain.revues.org/3074.

2 Voir Bogumil Jewsiewicki, "Mémoire et représentation pour un vivre ensemble ", EspacesTemps, n $84-85-86,2004$, p. 187-192, p. 188.

3 Ibid.

4 Je me réfere ici au concept de "groupalité » (groupness) définit par Brubaker en s'appuyant sur les travaux de Tilly comme résultant du sentiment d'appartenance à groupe produit par le partage d'un attribut catégoriel et de l'insertion effective dans ses réseaux de sociabilité. Au contraire de Tilly, Brubaker précise cependant que l'insertion dans les réseaux de sociabilité, si elle est essentielle dans le déclenchement des actions collectives, peut être minimale ou n'est même pas 
lien et le groupe. La narration ne se limite en effet pas aux éléments du récit au sens restrictif du terme. Au contraire, ainsi que l'a montré Gérard Genette, la distinction classique posée par Aristote entre le récit et la représentation, entre la narration et le dramatique, ne tient pas ${ }^{5}$. Comme la littérature ne peut représenter que par l'intermédiaire de la mise en récit, il en résulte que représentation (la mimesis) et narration (diegesis) sont équivalentes ${ }^{6}$. C'est en sens que Paul Ricœur exprime la dimension narrative de l'existence : la narrativité doit se comprendre à la fois comme action de mise en représentation et une assignation de sens, conçue dans la " mise en intrigue » de l'expérience humaine ${ }^{7}$. Cet emplotment caractéristique de la construction du récit se faisant toujours en référence à ce que Francis Farrugia définit comme des "arrières-plans ", des "récits fondateurs" qui s'enracinent dans des "narrations sociales imbriquées et réitérées (...) [lesquelles] nous fabriquent donc une mémoire et une sensibilité durables qui structurent notre identité, et déterminent organiquement, émotionnellement et intellectuellement notre rapport au monde ${ }^{8}$. Il s'agit alors, comme y invite l'auteur, de saisir la généalogie de la construction narrative de la conscience sociale du lien opérant entre l'individu et le collectif.

À partir de mon travail de recherche mené auprès de jeunes militants d'un parti politique libanais contemporain, le Courant Patriotique Libre (CPL), je me propose donc d'entreprendre ce travail d'archéologie de la mise en narration de l'attachement au groupe, afin d'interroger son rôle dans la construction et le maintien des groupes sociaux, mais surtout de montrer comment le concept d'identité narrative - défini comme produit par la projection de soi au sein de narrations sociales ${ }^{9}$ - permet in fine de venir réinterroger les fondements des collectifs que constituent les appartenances politiques et religieuses au sein de la société libanaise, marquée par de profondes divisions héritées tant de l'histoire des relations entre les segments sociaux que des cadres politico-juridiques qui les organisent ${ }^{10}$. Il faut ici préciser qu'au Liban, les principaux partis politiques,

toujours nécessaire pour la construction d'un sentiment de groupalité, c'est-à-dire un sentiment d'identification fort. Voir Rogers Brubaker "Au-delà de l'identité ", Actes de la recherche en sciences sociales, vol. 139, septembre 2001, p. 66-85, p. 79 ; voir également Charles Tilly, From mobilization to revolution, Reading, Addison-Wesley, 1978, p. 62 et suivantes.

5 Voir Gérard Genette, Figures II, Paris, Seuil, 1969, p. 55-56.

6 Ibid.

7 Voir Paul Ricœur, Du texte à l'action, Essais d'herméneutique II, Paris, Seuil, 1986.

8 Francis Farrugia, "Le syndrome narratif : théorie et terrain ", Cahiers internationaux de sociologie, $\mathrm{n}^{\circ} 127,2,2009$, p. 269-289, p. 273.

9 Sur le concept d'identité narrative, voir Margaret Somers, "The Narrative Constitution of Identity: A Relational and Network Approach ", Theory and Society, vol. 23, n 5, octobre 1994, p. 605-649.

10 La division de la société libanaise entre les groupes confessionnels qui la compose est envisagée ici dans une perspective constructiviste, comme étant le produit d'un processus d'institutionnalisation entendu comme "routinisation " des modes d'interactions dans un contexte spatiotemporel donné (voir Peter Berger \& Thomas Luckmann, The social construction of reality, 
dont le CPL, fonctionnent avant tout comme des " sociétés partisanes " ${ }^{11}$, c'està-dire qu'ils se présentent comme l'incarnation de sous-univers de signification coexistant et interagissant au sein de l'espace libanais. L'affiliation politique se comprend avant tout comme une inscription de soi dans une " manière d'être et de vivre $»^{12}$.

Au cours de mes rencontres avec les jeunes du Courant Patriotique Libre, cette insertion au cœur de l'univers partisan s'est manifestée par une récurrence de références, de dates, d'événements et de personnages qui, mobilisés par mes interlocuteurs pour dire leur lien au parti dans le cadre de l'interaction de recherche ${ }^{13}$, ont composé en quelque sorte un roman partisan, un refrain revenant invariablement et de manière régulière, tant au cours d'un même entretien qu'entre les différentes rencontres, bien que sous des formes différentes. En explorant cette diversité des " traductions " individuelles du récit partisan et partant de l'hypothèse que la structuration de l'univers de signification en vigueur au sein d'une société partisane comme celle du CPL se fait largement en référence à ces narrations sociales censées offrir une définition de la réalité sociale et des identités qui l’accompagnent ${ }^{14}$, je tâcherai de

Harmondsworth, Penguin Books, 1966, p. 77). Cette institutionnalisation des différenciations entre les groupes s'est vue renforcée au cours de l'histoire moderne du Liban. Tout d'abord, du fait du système politique qui organise la division du pouvoir entre les différents groupes confessionnels faisant que toute revendication politique se trouve presque systématiquement traduite en termes communautaires ; ensuite à cause de l'ordre juridique qui assigne à chaque Libanais un droit personnel spécifique en fonction de son appartenance confessionnelle, la catégorisation sociale est donc d'autant plus prégnante qu'il est pratiquement impossible de passer d'un groupe à un autre, si ce n'est par conversion religieuse ; enfin, les divisions se sont trouvées renforcées de par les conflits qui ont jalonné l'histoire des relations entre les groupes, en particulier la guerre civile de 1975-1990. De fait la question de l'appartenance devient l'élément central dans un grand nombre d'interactions sociales.

11 Voir Franck Mermier \&Sabrina Mervin (dir.), Leaders et partisans au Liban, Paris-Beyrouth, Karthala-IFPO-IISMM, 2012.

12 La formule est de Jean-François Bayart in Matière à politique, p. 218. Ainsi, Naim Qassem définit l'adhésion au Hezbollah, sans doute le plus connu des partis libanais, comme l'appartenance à " une Nation" : " ceux qui adoptent les objectifs du parti (...). Les affiliés n'étant pas les seuls à participer aux buts du parti. La définition de l'affiliation au parti n'est pas liée à une carte (...) " (in Hizbullah. The story from within, Londres, Saqi, 2005, p. 59-60). De la même manière, si le CPL a lancé depuis plusieurs années une campagne "d'encartement " de ses membres actifs, le groupe auquel le parti se réfère en tant que sa base sociale va bien au-delà de la minorité détentrice des cartes d'adhésion officielles. De ce fait, les réalités sociales pouvant se rattacher à l'ordre des sociétés partisanes apparaissent souvent multiples.

13 Par soucis de concision, et parce que cette contribution entend souligner avant tout les structures des récits des militants en eux-mêmes, les extraits d'entretiens sont livrés ici sans mise en contexte approfondie. Le retour analytique sur les conséquences de l'interaction de recherche dans la production des données sera ponctuellement explicité et ne fera donc pas l'objet d'un traitement systématique qui pourrait alourdir la démonstration.

14 Chaque réalité sociale intègre une certaine définition de l'identité de ses membres. Voir Peter Berger \& Thomas Luckmann, The social construction of reality, op. cit., p. 196. 
montrer comment, dans ces témoignages, sont convoqués les divers arrières plans du processus d'identification avec le CPL. Pour ce faire, je m'appuie sur un corpus d'une centaine d'entretiens réalisés auprès de militants et sympathisants du CPL au cours de mon travail de thèse entre l'automne 2007 et le printemps $2011^{15}$, complétés par des observations minutieuses ainsi que par nombre de sources officielles du parti (publications, brochures, site Internet du mouvement, etc.).

Je m'intéresserai dans un premier temps à l'organisation de la narration partisane, structurée autour d'une mise en intrigue spécifique et de personnages centraux (1). Je montrerai dans un deuxième temps comment cette narration est multi-vocale, convoquant diverses échelles. Celles-ci ordonnent des mémoires collectives ici définies, dans la lignée des travaux de Halbwachs ${ }^{16}$, comme des cadres référentiels socialement construits agençant les expériences et s'exprimant dans les témoignages au travers de structures narratives identifiables. Narration, mémoire et lien social étant donc, dans cette perspective, intrinsèquement liées (2). Enfin, en guise de conclusion, l'exploration de ces dimensions permettra d'effectuer un retour réflexif sur la nature du lien politique au Liban (3).

\section{La mise en intrigue du récit partisan}

Le Courant Patriotique Libre puise son origine dans l'un des ultimes bouleversements survenus au cours des guerres libanaises ayant fait rage entre 1975 et 1990 : la nomination, en 1988, du commandant en chef de l'armée, le général Michel Aoun, à la tête d'un gouvernement de transition, composé exclusivement de militaires ${ }^{17}$. Cette désignation, décidée dans les derniers instants de son mandat par le président sortant, Amine Gemayel, ne fut guère favorablement accueillie, tant sur la scène libanaise que régionale. Pourtant, prônant inlassablement son slogan "Liberté, Souveraineté, Indépendance "

15 Il s'agit d'entretiens semi-directifs réalisés auprès d'étudiants, de sympathisants et de cadres du mouvement. Comme mentionné précédemment, la possession d'une carte de membre ne constituait pas un critère de sélection. Les jeunes rencontrés l'ont été parce qu'ils se définissaient eux-mêmes comme attachés à ce mouvement et participaient, à des degrés divers et de manière plus ou moins soutenue, à ses activités. Nous avons donc retenu une définition par l'action et la définition de soi au détriment d'une définition légale qui, dans le cas libanais, ne parait pas permettre de rendre compte du phénomène partisan.

16 Voir en particulier Maurice Halbwachs, La mémoire collective, Paris, Seuil, 1997.

17 Le cabinet dirigé par Michel Aoun se compose théoriquement des six membres du conseil de guerre, constitué de généraux représentant les six communautés les plus importantes du Liban (les Sunnites, les Chiites, les Druzes, les Maronites, les Grecs-Orthodoxes et les GrecsCatholiques). Toutefois, soumis à de fortes pressions, les trois membres des communautés musulmanes se retirent. Seuls les généraux Issam Abu Jamra (Grec-Orthodoxe) et Edgar Maalouf (Grec-Catholique) forment l'équipe de Michel Aoun (lui-même représentant les Maronites). 
[Hurriyyeh, Siyyâdeh, Istiqlâl], Michel Aoun parvint à mobiliser une frange importante de la population vivant dans les territoires sous son contrôle, à majorité chrétienne. De nombreuses manifestations furent alors organisées autour du palais présidentiel de Baabda en soutien au "Général ». Fort de cet appui, Michel Aoun avait lancé un double combat, face à l'occupation syrienne du Liban et contre l'hégémonie milicienne qui y régnait. Élément perturbateur du jeu libanais, le général fut finalement renversé par une opération militaire syrienne, le 13 octobre 1990.

Dans la république de Taëf qui fit suite aux années de guerre, les militants " aounistes " s'organisèrent progressivement et menèrent à partir du milieu des années 1990 des manifestations sporadiques, plus ou moins encadrées par des directives de Michel Aoun lancées depuis Paris où il a trouvé refuge avec son entourage. Les militants de ce qui devint officieusement le CPL à la fin des années 1990 prirent de plus en plus de visibilité en dépit de la forte répression s'abattant ponctuellement sur eux, et participèrent largement aux " printemps de Beyrouth » en 2005, qui aboutit au départ des troupes syriennes du Liban. Michel Aoun rentra alors d'exil, le 7 mai 2005, et organisa son courant en parti politique. En février 2006, le CPL signa une feuille d'entente avec le mouvement chiite du Hezbollah et rejoignit les forces d'opposition à la coalition pro-occidentale du 14 Mars, menées par le Mouvement du Futur de la famille Hariri et incluant l'ancienne milice chrétienne des Forces Libanaises, à laquelle l'armée du Général s'était violemment opposée en 1989 et 1990. Après avoir participé à un gouvernement d'union nationale entre 2008 et 2011, le CPL s'empara avec ses alliés de la majorité parlementaire ouvrant la voie au contrôle du gouvernement en janvier 2011.

Cette histoire du mouvement, ici brièvement exposée, joue sans surprise un rôle central dans la socialisation partisane. En témoigne les propos d'un dirigeant de l'éducation politique au sein du CPL retraçant l'itinéraire de la transformation du mouvement en parti politique :

Par où commencer? Par la formation! (...) Il faut créer une base. La culture à lui dispenser, elle est déjà diffuse, dans nos pratiques (...). Certains [militants] avaient déjà été engagés dans la lutte mais sans trop réfléchir sur la signification de ce quils faisaient, d'autres également étaient loin, il fallait leur faire connaître le passé, l'histoire du courant. Il fallait donner sens à tout ça. Donc il fallait mettre au clair l'identité du CPL : qui est ce CPL, quel a été son combat, ses origines, quels sont ses symboles. ${ }^{18}$

Dans l'objectif avancé pour cette formation interne, "regrouper, rassembler, rassurer, faire face ", donner un sens à l'histoire du mouvement et à celle de son combat apparait essentiel. Cette signification s’appuie sur une série de dates clés,

18 Entretien avec le responsable du comité d'éducation politique du CPL, le 27 avril 2008. 
mises en avant par le discours public de l'organisation ${ }^{19}$. Parmi ces dates, les plus importantes demeurent le 14 mars 1989 (proclamation par le général Aoun de la " guerre de Libération » contre la présence armée syrienne), le 13 octobre 1990 (la chute du Général), le 7 août 2001 (arrestation des principaux cadres du mouvement clandestin par les autorités libanaises soutenues par Damas), et le 7 mai 2005 (retour du Général à Beyrouth) rebaptisé "Orange Day », du nom de la couleur fétiche du mouvement, par les jeunes activistes dans les universités. Interroger ${ }^{20}$ les militants sur le sens que revêtent pour eux ces moments scandant l'histoire partisane permet de révéler le travail d'ordonnancement des souvenirs et des représentations effectué par le biais du récit d'organisation.

14 mars 1989 :

À mon avis, c'est vraiment le début de la révolution contre la Syrie. Voilà. En fait, les gens oublient cette date, comme si ça ne voulait rien dire. Alors que vraiment, cétait au moment où les choses allaient le plus mal que les gens ont fait la révolution. Les Syriens n'étaient pas encore partout, ils ne contrôlaient pas tout... c'est là que la révolution s'est faite, mais c'est là que le monde nous a abandonnés (...). C'est aussi là que certains Libanais nous ont abandonnés. Pour de l'argent ou du pouvoir, ils ont oublié leur pays et nous ont vendus à la Syrie (...). (Johnny, étudiant, militant étudiant au sein de l'Université Saint-Joseph, né en 1989, entretien le 29 octobre 2008)

Ça représente le jour où le Général a annoncé la guerre contre les troupes syriennes en 1989. (...) C'était la première fois que quelqu'un avait osé dire non contre les projets que le monde entier et les grandes nations préparaient pour nous. Ça représente un jour où, chaque année depuis 1990, les jeunes descendaient dans la rue pour défier les forces de sécurité libanaises et syriennes (...). Je me souviens particulièrement du 14 mars 2004, (...) il y avait aussi des gens des Forces Libanaises qui participaient mais ils se sont retirés car (...) ils avaient passé un deal avec le pouvoir. Je me souviens très bien de ce jour. Et il y a bien sûr le 14 mars 2005. Ça a été comme sills avaient arraché de ce jour son esprit pur. Ils étaient venus le 14 mars 2005 avec l'idée que nous représentions la principale force [d'opposition à la présence syrienne] et puis dès le 15 mars ils ont altéré tous ces principes quincarnaient pour nous le 14 mars. C'était comme si le 14 mars on croyait que l'on avait arraché les autres vers nos principes, mais non, avec le temps on a bien vu que ce n'était pas ça. Ce n'était qu'un jour de plus de déception et de mensonge. (Édouard, étudiant, militant du CPL au sein de l'Université Américaine de Beyrouth, né en 1990, entretien le 29 octobre 2010)

19 Au-delà de la référence dans les discours ou matériaux (tracts, etc.) du parti, le CPL a développé un appareil et des techniques de communication. Il organise des commémorations, produit, via sa chaine de TV des documentaires ou des reportages consacrés aux événements, enseigne leur déroulement lors des formations, marque leur anniversaire sur la page Internet du parti par l'ajout de symboles ou de slogans spécifiques.

20 Suite à une série d'entretiens exploratoires au cours desquels il était demandé aux militants de citer les événements majeurs de l'histoire libanaise, j'ai établi un protocole d'entretiens fondé sur une double rencontre. La première prenait la forme d'un entretien semi-directif classique, destiné à retracer la trajectoire biographique et explorer les représentations des acteurs. La seconde se fondait au contraire non sur des questions mais sur les réactions des enquêtés confrontés successivement à des dates et des photos de personnages. 
Présenté comme le point de départ de l'aventure " aouniste ", le 14 mars 1989 renvoie pour les militants à un temps du Paradis perdu, celui au cours duquel les Libanais prirent conscience de leur situation et décidèrent de changer le cours de leur destin. Les récits faits de la période de liesse populaire entourant l'avènement du Général, devenu l'incarnation du "Liban Libre " ${ }^{21}$ et du "Refus chrétien ${ }^{22}$ sur une scène politique meurtrie par les années de guerre et de division, sont nombreux. Dans le récit partisan, le sens de l'événement est précisément l'union du peuple contre la conspiration des ennemis du Liban, qu'il s'agisse des puissances internationales ou des milices régnant sur les micros-territoires arrachés à l'autorité d'un État qui n'existait plus. En dépit de l'espoir suscité par l'irruption de ce temps nouveau, la déception et la trahison qui s'annoncent affleurent déjà.

Mais, comme en témoignent les deux extraits présentés ici, le 14 mars 1989 n'acquiert sa pleine signification que dans le contexte présent du temps de l'énonciation du récit. L'événement est alors construit en contre-type du 14 mars 2005, date d'une manifestation monstre regroupant près d'un million de Libanais sur la place des Martyrs de Beyrouth pour demander le retrait des troupes syriennes présentes sur le territoire national depuis 1976. Acteur majeur de la mobilisation, le CPL, qui n'était alors qu'un mouvement peu structuré et dont le chef se trouvait encore en exil, fut progressivement évincé par les forces l'ayant rejoint dans sa contestation de la présence syrienne et qui prendront d'ailleurs le nom de coalition du 14 mars. Dans l'optique du parti aouniste, le rappel du 14 mars 1989 intervient donc comme un moyen de saper la légitimité de ses adversaires politiques. Pour les militants, dénoncer la tromperie du 14 mars 2005 et l'opposer à l'authenticité du 14 mars 1989 et des mobilisations commémoratives l'ayant suivi permet donc de se positionner sur l'échiquier politique présent et plus généralement dans la société libanaise par opposition aux autres groupes partisans, notamment aux Forces Libanaises.

\section{3 octobre 1990 :}

Je me rappelle bien du 13 octobre 1990, ça m’a touché énormément et je ressens encore maintenant ce que j'ai ressenti ce jour-là. J'étais dans mon village qui était déjà occupé par les Syriens, depuis 1975. Je me rappelle que ma mère nous a réveillées (...) le matin pour qu'on descende se réfugier chez les voisins qui avaient une petite cave. C'était parce que les Syriens bombardaient Baabda. Nous sommes restées

21 Titre d'une chanson composée par Guy Béart, qu'il viendra interpréter à Beyrouth en 1989, mais aussi d'un ouvrage publié par Salvatore Lombardo, journaliste français soutenant comme nombre de ses confrères, de personnalités politiques, d'intellectuels et d'écrivains, la cause du Général qui suscita un grand engouement en France. Pour un aperçu de ces soutiens, voir notamment : Salvatore Lombardo, Liban Libre, Marseille, Éditions Transbordeurs, 2005 ; Daniel Rondeau, Chroniques du Liban rebelle, 1988-1989, Paris, Grasset, 1991 ; Jean-Paul Bourre, Génération Aoun. Vivre libre au Liban, Paris, Robert Laffont, 1990.

22 Titre d'un article publié par l'historienne libanaise May Davie, " Aoun ou le refus chrétien. Liban : octobre 1989-janvier 1990 », Temps Modernes, p. 536-537, 1990, p. 147-180. 
jusquà 9h du matin, puis notre voisin, qui était des Forces Libanaises, est descendu (...) et il sest moqué de la chute du Général. Moi, je ne le croyais pas... je suis montée chez nous pour entendre la radio... je me rappelle bien que jai pleuré. Il y avait des Jeeps syriennes qui passaient dans le village et c'était la première fois que je me sentais tellement incapable de faire quoi que ce soit, rien, vraiment incapable... alors j'ai pris un caillou, et je l'ai jeté vers les Jeeps syriennes, et je pleurais, je pleurais.. c'était vraiment décevant. (Lise, journaliste, cadre du CPL, née en 1982, entretien le 10 novembre 2007)

C'est le pire scénario... c'est la mort... mais aussi la renaissance, comme dans la légende d'Adonis, la résurrection, comme Jésus Christ. On était battus mais on allait continuer la lutte et accomplir notre but. (...) Mais en ce jour-là, je me souviens des avions syriens, les Sukhoïs, qui venaient des montagnes, de la chaîne Est comme on dit ici au Liban, pour bombarder le palais présidentiel de Baabda et le ministère de la défense. C'est (...) une défaite complète... le discours du Général qui capitule, assiégé... c'est le jour des martyrs qui sont restés au combat jusqu’à la mort (...). (Pierre, journaliste, cadre du CPL dans la région de Zahlé, né en 1981, entretien le 6 mars 2009)

C'est comme un phonix, l'oiseau, qui renaît de ses cendres... le CPL aussi a continué. Il a commencé le 14 mars et là, il a survécu. C'était un coup violent, militaire et politique, mais on a trouvé quelques âmes qui ont continué l'esprit du Général, incarné en eux... ils ont continué la lutte contre l'occupation syrienne...

- Tu te rappelles du jour?

Non. Je ne me rappelle pas du jour mais je me rappelle du visage de mon père qui pleurait. J'avais alors six ans et quand je l'ai vu pleurer, j'ai pleuré aussi... il m'a dit: "Le Liban est perdu». Quand tu me dis 13 octobre 1990, je fais un flash-back sur les photos des soldats qui ont donné tout ce quils pouvaient, qui se battaient avec tout ce qu'ils trouvaient parce qu'il n'y avait plus d'armes, rien. Je me souviens du discours du Général quand il a demandé le repli et donné le commandement à Émile Lahoud. C'est tout [silence]. (Georges, ancien étudiant de l'Université saint-Joseph, militant du CPL, né en 1984, entretien le 30 avril 2008)

La Chute. L'expérience négative qui entraine la disparition du réel tel que les partisans du Général l'avait jusqu’alors construit. Les témoignages des jeunes militants démontrent clairement comment la mémoire collective de l'organisation délimite une stricte signification à l'événement : l'anéantissement du mouvement populaire dans lequel les irréductibles puiseront la force de leur résistance. Si chacun tend à se situer au moment de l'événement, les canons du récit édictés au sein du collectif partisan se retrouvent dans la majeure partie des évocations : l'insistance sur la disproportion des forces évoquée par l'utilisation de l'aviation syrienne (l'unique fois où les Syriens seront autorisés à utiliser leur forces aériennes dans le conflit libanais), l' allocution radiodiffusée du Général qui, pour épargner son peuple, met fin aux combats et, bien sûr, la nécessaire continuation de la lutte en dépit de l'effondrement du rêve ainsi que le culte des martyrs. De la sorte, en offrant un cadre pour l'appréhension de l'expérience biographique de chacun, la mémoire partisane de l'événement fondateur rend possible la projection de soi dans l'univers de sens du groupe, illustrant un processus d'attachement fondé sur le partage d'une lutte politique commune. 


\section{7 août 2001 :}

C'est une date importante pour le CPL. C'est le jour où on a emprisonné tous les responsables du parti, y compris le général Nadim Lteif (...) qui est un général de la Police. Il a ensuite été battu par des gardes. Ce jour, nous avons vu une réaction énorme du peuple libanais, nous avons démontré à tout le monde, le 7 août puis le 9, que la cause de la libération du pays n'est pas oubliée... et ça a eu beaucoup de conséquences au Liban et dans le monde, ça a montré la tyrannie syrienne. Il y a une image (...) qui est très touchante: une sæur qui tentait de protéger son frère des coups. Et elle a été battue aussi. C'était des Libanais pas des Syriens! Cette photo est devenue la photo officielle de tous les petits agendas du CPL depuis 2001 jusqu'à aujourd'hui. Sur la première page, vous pouvez voir la photo de ce jeune homme penché au sol et que les services de renseignements frappaient, avec sa sour à ses côtés... (Hani, étudiant, militant du CPL au sein de l'Université saint-Joseph, né en 1985, entretien le 30 avril 2008)

C'est une des étapes que les jeunes du CPL devaient accomplir. C'était comme un carême si tu veux. C'était un honneur pour eux de faire cela quand ils étaient les seuls à faire cela, les seuls à lutter contre l'occupation syrienne. On voit aujourd'hui les hommes au pouvoir, les gens du Futur et du PSP [deux des partis principaux de la coalition du 14 Mars à l'époque], essayer de nous faire croire que le 7 août, c'est eux. Ils volent cette date. Le 7 août ressemble un peu au 13 octobre, mais politiquement. (...) J'étais là-bas moi, sur le terrain, lors de la deuxième manifestation du 9 août. Mais quandjai vu ce qui se passait, je me suis réfugié dans une poubelle. Quand je suis rentré à la maison, je suis resté toute la journée dans ma chambre à pleurer parce que deux de mes amis proches ont été arrêtés. J'étais descendu seul, sans le dire à personne. Quand jai vu ce qui se passait, j'ai reculé et je me suis caché. (...) J'étais jeune, c'est resté dans mon coeur jusqu'à maintenant. En plus, mes camarades continuent de temps en temps de se moquer de moi avec ça, ils n'appellent "Jabân [lâche]". (Georges, entretien le 30 avril 2008)

Le 7 août symbolise le moment de transition, la période de lutte censée aboutir au Salut de la cause. Cette phase est marquée par l'apparition de personnages incarnant la figure idéale du militant, héros montrant la voie à suivre et, en ce sens, véritables prophètes de la libération à venir. Les témoignages reproduits montrent la manière dont la socialisation partisane utilise ces personnages pour les présenter comme des phares éclairant la conduite des autres militants via la fabrication d'icônes telles que ces photos reproduites sur les calendriers édités par le collectif. Le récit de Georges illustre a contrario le poids normatif exercé par ces modèles sur le comportement attendu des jeunes Aounistes. $\mathrm{Ne}$ pas s'être fait arrêté ni malmené par les forces de sécurité devient synonyme de lâcheté et objet de quolibets de la part de ses camarades au point qu'y compris lors de l'entretien, il se sent obligé de justifier son comportement en évoquant son jeune âge. Plus que l'acte de fuite, c'est le non-partage de l'expérience de ses camarades qui perturbe Georges, coupable d'avoir failli à se conformer aux idéaux que son organisation lui enjoignait de suivre.

7 mai 2005:

Moi, jai un regret ce jour-là, car je n'ai pas pu arriver au centre-ville. Je suis parti un peu tard de la maison et j'ai passé la journée sur la route, coincé dans les embouteillages. C'est un regret, dommage. Le retour du Général, et je suis sûr que tu sais 
déjà qu'entre 1990 et 2005, les partisans du CPL s'efforçaient d'écrire sur les murs "Aoun revient». Et Aoun est revenu. C'était un jour grandiose, une liesse populaire indescriptible. Il n'y a jamais eu une manifestation aussi grande pour un seul parti ou une seule personnalité. C'était quelque chose de jamais vu dans l'histoire du Liban et ça rappelait beaucoup Baabda [les mouvements populaires autour du palais présidentiel] de la période 1988-1990. Quand le Général a prouvé dès la première minute qu'il n'avait pas changé, que son tempérament était le même, avec sa phrase légendaire : "Yầ shaab Lubnân al-'azîm [Oh grand peuple du Liban] " qui a eu le même effet quà Baabda en 1989. (Hugo, cadre au sein de la section estudiantine du CPL, né en 1988, entretien le 23 octobre 2010)

"'awdat al-watan ila-l-watan [le retour de la patrie (terme également utilisé au Liban pour désigner un soldat, membre de l'institution nationale par excellence, lorsqu'on s'adresse à lui) à la patrie] ", tu connais cette formule? Là, j’ai beaucoup pleuré (...). Le 7 mai c'est toute une nouvelle phase qui a commencé pour moi... je me suis dit maintenant, je peux aller où je veux pour continuer mes études, mais avant je ne voulais pas quitter le Liban pour pouvoir lutter pour ça... mais maintenant cétait un moment d'émotion parce qu'on ne croyait pas qu'il allait revenir. Toutes les déceptions quion a vécues depuis le 13 octobre, le 7 mai 2005, c'était vraiment le contraire: toute la joie, toute la liberté, tout ce pour quoi on a lutté pendant quinze ans (...) ça c'était la récompense. (Anis, étudiant en doctorat au sein de l'Université Libanaise, militant du CPL membre du comité d'éducation politique, né en 1975, entretien le $1^{\text {er }}$ décembre 2009)

Le 7 mai équivaut à un apogée, une réminiscence du Paradis originel de la période 1988-1990 mais aussi la clôture d'une phase de l'histoire de l'organisation. Le retour du Général vient marquer la Résurrection promise le jour du 13 octobre. Ce pinacle de l'œuvre militante marque aussi un tournant dans les expériences biographiques en ouvrant le champ des possibles pour des activistes longtemps focalisés sur un seul et unique but. Le récit ainsi conté, en se construisant en référence à un ensemble de codes partagés, assure une des conditions de possibilité de l'existence du groupe partisan en tant que groupe social : " la faculté de remonter le cours du temps et de repasser sans cesse sur les traces qu'[il] a laissées de [lui]-même ${ }^{23}$. L'événement signe surtout le retour du personnage principal de la narration partisane, Michel Aoun. Au cours de mes rencontres avec les militants, le leader est le plus souvent défini selon un triple champ lexical : celui de l'héroïsme, à mettre en rapport avec la mise en intrigue de l'Histoire, mais aussi celui de la famille - il est le père, puis le grand-père pour les générations successives de ses partisans - et enfin celui de la prophétie - le chef est un visionnaire guidant son peuple. Cette pluralité des espaces de représentation du leader en fait le symbole d'un groupe organique, un entre soi familial, uni au sein d'une même narration. Elle fonde également une généalogie symbolique, constituant les nouveaux adhérents en successeurs reprenant le flambeau de combats passés.

23 Maurice Halbwachs, La mémoire collective, op. cit., p. 192. 
Les récits construits par les militants autour des moments clés de leur collectif, apparaissent ainsi non seulement fondamentaux dans l'histoire du mouvement, mais ils se trouvent attachés par une mise en intrigue spécifique, les reliant entre eux ainsi qu'au temps présent au fil d'une description de la temporalité épousant les étapes successives d'une expérience collective. Si le sentiment de partager une histoire commune forme une caractéristique classique de l'attachement au collectif, s'interroger sur le mode de constitution de cette représentation du temps constitue un détour indispensable pour comprendre les fondements du lien au sein du groupe. L'histoire collective est donc avant tout produite par un emplotment spécifique. Cette mise en intrigue qui opère ici selon le modèle Paradis perdu - Chute - Purgatoire - Résurrection rappelle la structure des mythes judéo-chrétiens, telle que décrite par Genette dans son analyse du temps proustien ${ }^{24}$. Un parallèle qui, en plus des nombreuses références contenues dans les discours des acteurs, inciterait à penser que l'on ne peut comprendre pleinement la construction de ce récit aouniste et donc du lien qu'il représente qu'en le mettant en rapport avec des arrières plans pluriels.

\section{La multi-vocalité de la mémoire dans les récits biographiques}

S'ils révèlent la mise en intrigue de l'histoire perçue dans le cadre partisan, les récits des militants témoignent avant tout d'une construction de leur attachement au collectif. Au cours de la situation d'entretien qui agit comme tout cadre d'interaction, ces jeunes, interrogés parce quills se disent adhérent du CPL, livrent pour décrire leur appartenance un récit unifié par des interprétations communes de l'histoire et de la réalité. Interprétation est ici à comprendre au double sens de donation de signification et manière d'incarner un personnage ${ }^{25}$. Les caractéristiques de ces personnages sont elles-mêmes définies en regard de représentations au sujet de l'idéal-type du militant, construit en référence à des héros au premier rang desquels se trouve le leader du mouvement. Leurs récits biographiques sont donc des actes de mise en représentation de leur attachement au groupe. Mon objectif étant de comprendre les processus par lesquels le lien est construit et représenté par le recours à une identité construite dans la narration, il est possible de dégager deux axes principaux : la manière dont leurs récits produisent leur positionnement en tant que membre du groupe d'une part, et, d'autre part, les arrières plans permettant de déconstruire leur représentation de leurs expériences.

La notion de position empruntée à Rom Harré puise son origine dans la volonté de rendre plus effective la notion de rôle pour l'étude des interactions. Contrairement au rôle, défini de manière stable, une position est fluide, créée

24 Voir Gérard Genette, Figures I, Paris, Seuil, 1966, p. 65.

25 Voir Francis Farrugia, "Le syndrome narratif : théorie et terrain ", op. cit., p. 270. 
dans l'interaction discursive. Elle définit la localisation des acteurs dans la structure narrative, ouvrant la voie à un répertoire de significations mobilisables en situation. Une position est donc un processus d'attribution intersubjective de sens dans l'interaction participant à la définition de la réalité telle que perçue par les acteurs ${ }^{26}$. Elle se réalise concrètement dans la dimension dramatique de la narration dans la mesure où elle rend compte de la manière dont un acteur cherche à occuper un espace déterminé et à incarner un personnage défini par une localisation relative et une ligne de discours qui lui est attachée.

Dans leurs récits, les jeunes Aounistes construisent discursivement leur positionnement non seulement face au chercheur, mais aussi dans la structure sociale qui les entourent. Signaler comme le fait Johnny que certains ont oublié le sens du 14 mars 1989 signifie qu'il appartient au groupe qui ne l'a pas fait, lui ouvrant la voie à un répertoire de dénonciation morale de ses adversaires corrompus par l'argent ou le pouvoir selon ses dires. De même, dans l'extrait suivant, Édouard oppose les militants du CPL à ceux de leur rival des Forces Libanaises (FL) qu'il positionne dans son récit de la manifestation du 14 mars 2004 comme des traitres ayant pactisé avec le pouvoir. Ce faisant, il revendique sa légitimité et celle de son parti à faire l'exégèse de la réalité. Leurs évocations du 14 mars fonctionnent donc comme un acte de positionnement tant personnel (se revendiquer comme membre du CPL) que collectif (situer leur parti dans la structure politique présente). De même, dans le récit de son expérience du 13 octobre 1990, Lise oppose son personnage ainsi que sa famille à un voisin hostile, soutenant le groupe antagoniste des FL. Au-delà d'une histoire partagée et de l'expérience d'émotions communes aux membres de la collectivité aouniste, Lise construit donc aussi une frontière. La dénonciation du « vol » du 7 août 2001 par les adversaires politiques du moment du récit fournit un autre exemple. Les positions sont donc construites au moment de l'acte d'énonciation du récit mais se structurent en référence à un temps autre, interne à la narration. S'ajoute que les éléments de discours mobilisés pour construire ces positions le sont dans la situation d'interaction avec le chercheur : il s'agit de revendiquer l'identification de soi comme un membre du collectif afin d'en apparaitre comme un porte-parole légitime.

Ces positions revendiquées par les activistes dans leur présentation de soi reposent sur des storylines spécifiques dont il s'agit de faire l'archéologie. Leurs récits se construisent dans des dialogues avec des cadres sociaux multiples. La narration de leur expérience se définit en référence à différentes échelles de signification. La première échelle correspond au temps biographique de l'acteur. Les souvenirs personnels et familiaux définis comme des mémoires

26 Voir Rom Harré, " Positioning theory ", 2004, en ligne : www.massey.ac.nz/-alock/virtual/ positioning.doc ; Rom Harré \& Luk Van Langenhove, Positioning Theory : Moral context of intentional action, Oxford, Blackwell, 1999. 
vives ${ }^{27}$ permettent l'inscription de soi dans une mémoire collective imaginée. Comme l'illustrent parfaitement les témoignages de Lise et de Georges sur le 13 octobre, ou d'Anis sur le 7 mai, l'émotion y joue un rôle central. On y voit comment le groupe aouniste s'inscrit dans des réseaux de relation avec d'autre collectif, ici les familles des militants, témoignage de la socialisation de ces jeunes dans un milieu qui lui-même s'inscrivait dans le mouvement aouniste. Les souvenirs contés prennent donc naissance dans les deux groupes, la famille et le parti, et cette convergence rend l'attachement plus for $\mathrm{t}^{28}$. Si la causalité introduite par le récit partisan fonde les rapports que les individus entretiennent avec leur souvenir, la mémoire collective trouve son nécessaire support humain dans cet ensemble de mémoires vives. Ainsi que l'a montré Maurice Halbwachs, les récits individuels représentent donc un point de vue particulier sur la mémoire collective du groupe ${ }^{29}$.

La deuxième échelle correspond au temps de l'organisation. Les nombreuses références aux différentes phases de l'histoire, à ses héros ou modèles, mais aussi à ses adversaires passés et présents dessinent l'image d'un groupe humain engagé dans un combat politique. Les récits biographiques ne prennent en effet sens que par rapport aux luttes originelles et actuelles que le collectif partisan mena sur le terrain et continue aujourd'hui de conduire. Ces combats désignent autant d'adversaires successifs : les forces syriennes, les milices et particulièrement les Forces Libanaises, les collaborateurs, puis les adversaires politiques d'aujourd'hui. La mémoire de ces luttes traverse les individus qui composent le collectif et continue d'en informer l'attachement comme en témoigne la forte charge morale associée à l'action militante que l'on retrouve notamment dans les récits portant sur le 7 août 2001. Le temps de la lutte armée contre la Syrie et les forces miliciennes reste également omniprésent, notamment au travers du souvenir des martyrs.

La place spéciale réservée à l'ennemi des Forces Libanaises révèle enfin la troisième échelle. Il s'agit d'une échelle de temps long, rattaché à un double référent spatio-communautaire. Outre la récurrence de référents symboliques tirés du Christianisme (carême, la Résurrection de Jésus Christ, etc.), la fréquence de la construction en miroir effectuée dans les récits militants entre les soutiens du CPL et les militants des FL est à resituer dans le champ d'expérience des populations majoritairement chrétiennes vivant dans « les régionsEst ", non-occupées par la Syrie avant 1990. Avec l'avènement du Général, ces régions ont été soumises à une intense lutte pour le leadership local entre

27 La notion de mémoire vive à propos des discours produits par les adhérents d'un groupe social défini fut notamment utilisée par Marie-Claire Lavabre dans son étude de la mémoire collective du Parti communiste français. Voir Marie-Claire Lavabre, Le fil rouge. Sociologie de la mémoire communiste, Paris, Presses de la FNSP, 1994, p. 24-25. 
l'armée censée incarner le retour de la légitimité de l'État que voulait imposer Michel Aoun et les Forces Libanaises. En outre, Michel Aoun ainsi que son rival Samir Geagea, chef des FL, sont de confession maronite. Le système politique libanais organisant la distribution des postes entre les sectes a donc contribué à nourrir la rivalité entre les deux formations ${ }^{30}$. Si de nombreux musulmans ont soutenu l'action du CPL et continuent de le faire, il n'en reste pas moins que le mouvement s'inscrit de par son implantation spatiale originelle et de par l'origine confessionnelle de son leader dans le long chapitre de l'histoire politique chrétienne au Liban. Au-delà d'une pluralité sociale et confessionnelle qu'il ne s'agit pas de gommer, les chrétiens libanais semblent en effet partager une perception de l'histoire les poussant à rechercher la sécurité face à un environnement musulman considéré comme menaçant ${ }^{31}$. Cette dimension spatiale et communautaire du mouvement aouniste se retrouve notamment dans le témoignage de Lise, évoquant " un voisin » des Forces Libanaises. Le fait qu'il s'agisse d'un voisin souligne l'imbrication territoriale mais aussi la proximité sociale des deux groupes rivaux s'opposant sur le modèle du factionnalisme : une logique de dualité, une opposition en miroir, et un jeu de miroir avec un troisième terme, qui serait dans le contexte régional la majorité musulmane ${ }^{32}$.

Les cadres collectifs ne sont par conséquent pas que des dates ou des personnages mais dessinent des courants d'expérience qui ont marqué les divers groupes sociaux au sein desquelles les acteurs s'inscrivent $t^{33}$. Les témoignages des militants s'expriment ainsi en référence à une pluralité de mémoires composant un dialogue à plusieurs voix (multiple voicing) ${ }^{34}$. Cette multi-vocalité donne corps

30 S'autoproclamant " patriarche de la rue » en référence au leadership politique exercé par le Patriarche de la communauté maronite, le leader du CPL semble prétendre jouer lui-même le rôle de représentant des chrétiens libanais. Propos tenus par Michel Aoun lors d'une interview télévisée, le 25 novembre 2007.

31 Voir Elizabeth Picard, "Les dynamiques politiques des chrétiens au Liban. Changement de statut et crise du leadership ", Monde arabe Maghreb-Machrek, n 153, 1996, p. 3-21, p. 5. Nourrie en particulier par le souvenir des violences intercommunautaires de 1840 et de 1860, cette aspiration explique largement l'insistance avec laquelle les élites chrétiennes s'attelèrent à intimement imbriquer la notion d'État libanais, l'identité spécifique libanaise (et particulièrement celle des maronites) et les garanties de leur propre domination politique. Pour une analyse de ce processus historique, voir Kamal Salibi, Une maison aux nombreuses demeures. L'identité libanaise dans le creuset de l'histoire, Paris, Naufal 1989.

32 Voir Isabelle Rivoal, «Division, équilibre, médiation. Une lecture de l'opposition factionnelle dans le monde arabe ", Ateliers d'anthropologie, n 27, 2004, en ligne : http://ateliers.revues. org/8649? lang=en.

33 Maurice Halbwachs, La mémoire collective, op. cit., p. 113.

34 Le concept de multiple voicing renvoie aux travaux de Mikhail Bakhtin. Voir Mikhail Bakhtin, The dialogic imagination: Four essays, Austin, University of Texas Press, 1981 [1930]; Speech genres and other late essays, Austin, University of Texas Press, 1986. Le concept est utilisé pour affirmer la pluralité des influences sociales contribuant à la production d'un discours. Voir, par exemple, Amy Shuman, "Entitlement and empathy in personal narrative ", in Michael Bamberg (dir.), Narrative - State of the art, op. cit., p. 175-184, p. 177. 
à une "combinaison mémoriale $"^{35}$ spécifique servant de base à l'identification avec le Parti. C'est le croisement entre cette mémoire collective imaginée et les mémoires vives reconstruites par la narration qui crée le lien unissant l'individu au groupe et vient représenter leur attachement. En ce sens, les témoignages présentés plus haut donnent à voir certaines versions particulières d'une narration sociale plus large fonctionnant comme un horizon au sein duquel chacun construit sa place ${ }^{36}$, offrant même à ceux qui n'ont pas de souvenir personnel un cadre pour se construire une vision du passé conforme à la mise en intrigue de l'histoire dans le récit partisan. Ce faisant, ils construisent comme réel par le discours une mémoire et une identité qu'ils lient et qui les lie au CPL. Le soi qu'ils présentent est alors en fait une conversation plutôt qu'un monologue. Il se forme dans le déroulement des interactions sociales, dans l'expérience de l'intersubjectivité. Le soi n'est donc pas une précondition de l'expérience, mais sa conséquence : c'est parce qu'ils se définissent comme des membres du groupe et sont insérés dans ses réseaux de sociabilités dont ils ont intégrés les normes, mais aussi parce qu'ils sont reconnus comme tels par autrui dans nombre de leurs interactions, qu'ils construisent leur soi en référence à ces cadres narratifs.

En déconstruisant le processus de formation de ces discours, on peut donc comprendre les ressorts de l'identification avec le parti : elle repose sur l'insertion au sein du cadre narratif partisan d'expériences tirées des multiples mémoires vives présentes chez l'acteur. La mémoire collective, garante du lien au sein du groupe ${ }^{37}$, est donc à concevoir comme une reconstruction narrative, composée de différentes strates spécialement agencées par l'acte de mise en discours. Cette énonciation se fait au temps présent de l'interaction et implique donc que la mémoire qu'elle dessine évolue en continu. Le groupe partisan n'est plus le même lorsqu'un étudiant né en 1990 s'y inscrit lors d'une rencontre avec un chercheur étranger qu'en 1989, au moment où les manifestations de soutien au Général se tenaient à Baabda. Si la mémoire collective entend " perpétuer les sentiments et les images qui forment la substance » du groupe et le distingue des autres ${ }^{38}$, le tableau qu'elle compose est nécessairement affecté par les conditions du temps de son énonciation. L'opposition à la coalition du 14 mars 2005, évoquée dans les souvenirs individuels ne prend de sens que dans la compétition politique présente qui voit s'opposer le CPL

35 L'expression est de Michel Verret, qui la définit à propos du Parti Communiste comme la réunion de la mémoire historique de l'organisation et des mémoires vives des individus qui la composent. Voir Michel Verret, "Mémoire ouvrière, mémoire communiste ", Revue Française de Science Politique, vol. 34, n 3, 1984, p. 413-427, p. 422.

36 Voir Gérard Lenclud, "S'attacher ", op. cit., p. 7.

37 À ce titre, Maurice Halbwachs affirme que la pérennité de la mémoire d'un groupe dépend de sa capacité à se concevoir en tant que tel lorsque les membres envisagent leur passé. Voir Maurice Halbwachs, La mémoire collective, op. cit., p. 58. Ibid., p. 139. 
à ses adversaires du moment. Mais le groupe ne peut exister que s'il prend conscience d'une histoire particulière qui, bien qu'en constante évolution, lui apparaît relativement stable.

Les récits des jeunes militants entendent ainsi non seulement les inscrire au sein du collectif et leur permettre de se revendiquer comme des membres légitimes par leur positionnement mais aussi affirmer leur symbiose avec la mémoire collective du CPL par la reprise de son interprétation dans la narration de leur souvenir. En suivant le fil narratif organisé en référence à l'histoire du parti et des populations qui le composent, leurs récits biographiques se posent comme mimétiques d'une histoire partagée, d'une expérience collective supposée commune de la réalité qui, bien que décrite comme la cause de l'attachement, en apparaît davantage comme la conséquence. Cette " mémoire collective » est une revendication de ceux qui se positionnent comme appartenant au groupe. Elle donne corps au groupe à partir du moment où les membres (auto-proclamés) utilisent le cadre de l'histoire du parti pour représenter leurs positions « d'attachés » dans les situations sociales. La mise en narration et l'identité narrative constituent donc un mode de réalisation du potentiel de la mémoire collective en interaction à partir d'une situation d'énonciation, sur la base de voix multiples traduisant des courants d'expérience divers dans lesquels l'acteur se positionne (personnel-familial, local, communautaire). En organisant ces courants par la mise en récit de la mémoire collective, les narrateurs réalisent un agencement spécifique de ces champs d'expérience et se posent à la fois comme les médiateurs, les acteurs et les auteurs de ce qui constitue la société partisane du CPL.

\section{Construire son identité, dire sa différence : le lien politique au Liban}

La représentation du lien dans les récits de ces jeunes Aounistes donne à voir comment la convocation d'une identité narrative permet la réalisation de cette combinaison d'échelles à base de la construction de communautés (au sens de groupes distingués les uns des autres) et leur perpétuation au travers d'une double mobilisation renvoyant aux deux dimensions de l'identité : inclusive (qui rassemble les membres du groupe) et exclusive (vis-à-vis de ceux qui n'en font pas partie). Ces identités narratives opèrent comme des " interprétations (à prendre au double sens de donation de signification et manière d'incarner un personnage) ${ }^{39}$. On comprend alors comment les schémas d'identification agissent via les narrations $s^{40}:$ " un homme, c'est toujours un conteur d'histoires, il vit entouré de ses histoires et des histoires d'autrui, il voit tout ce qui lui arrive à travers elles $»^{41}$.

39 Voir note 25.

40 Voir Rogers Brubaker, "Au-delà de l'identité ", op. cit., p. 76.

41 Jean-Paul Sartre, La nausée, Paris, Folio, 1972, p. 64. 
La manière dont les jeunes militants organisent leurs récits, le choix des souvenirs et des référents qu'ils convoquent pour représenter leur attachement au groupe permettent in fine de tirer quelques indications sur la nature du lien politique au Liban. Le CPL, à l'image des autres principaux partis du pays, apparaît constitué en tant qu'espace d'entre soi regroupé autour d'une mémoire commune construisant une frontière avec l'extérieur. L'identité narrative se déploie dans une histoire autoréférentielle qui nourrit l'attachement qu'elle a suscité. Ces histoires partisanes reposent sur des " mémoires privées " (private memory) $)^{42}$ éclatées et irréconciliables, d'autant plus hermétiques que les conflits passés y tiennent une place hypertrophiée, qui ont longtemps maintenu un "système d'amnésie collective " venu recouvrir, sous l'impulsion de l'État, les événements traumatiques des guerres civiles de 1975-1990 ${ }^{43}$. Ces frontières identitaires, sans cesse renouvelées par l'évolution des rapports entre les groupes cohabitant au sein d'une société imbriquée, opèrent comme vecteurs fondamentaux de l'attachement à un groupe.

Se concentrer sur la représentation narrative du lien permet en outre de voir comment les référents familial et communautaire continuent de nourrir le fonctionnement du collectif. Leur présence se trouve injectée indirectement, par le truchement des expériences sociales intégrées aux souvenirs des individus. C'est donc par le biais de la mémoire collective, une mémoire sociale et se vivant au présent, que les significations héritées de la famille et de l'appartenance confessionnelle réinvestissent le cadre partisan d'une organisation clamant pourtant sa volonté de dépasser les clivages familiaux, claniques et religieux. Tant le référent familial que le référent communautaire donnent une place centrale au leader, à la fois père (ou grand-père) et guide, qui se trouve par conséquent investi d'une légitimité incontestable et transcendantale. Le lien politique tel qu'il se constitue semble en fin de compte un lien existentiel constitutif du soi des adhérents dans au moins un triple processus d'identification : s'attacher, c'est se positionner comme membre du groupe dans les interactions au sein d'une structure sociale divisée ; s'attacher, c'est présenter une définition partagée de ce que le groupe et son histoire représentent tout en se plaçant sous l'autorité de son chef ; s'attacher, c'est enfin inscrire la définition de soi dans une mémoire collective multi-vocale.

Bruno Lefort

UMR 7310 IREMAM

IEP Aix-en-Provence

Tampere Peace Research Institute (TAPRI)

University of Tampere

bruno.lefort@uta.fi

42 L'expression est de Sune Haugbolle, "Public and private memory of the Lebanese civil war ", Comparative Studies of South Asia, Africa and the Middle-East, Duke University Press, vol. 25, n 1, 2005, p. 191-203, p. 199.

43 Ibid., p. 191. 


\title{
Résumé
}

La narrativité, parce qu'elle est à la fois action de mise en représentation et assignation de sens, constitue un des modes de construction et de représentation privilégié du lien social. Portant sur l'étude d'un groupe partisan libanais, le Courant Patriotique Libre (CPL), cette contribution mobilise le concept d'identité narrative d'abord pour décrire comment un groupe s'enracine dans une histoire spécifique conçue autour d'une mise en intrigue caractéristique. Il est ensuite établi que cette mise en intrigue s'appuie sur une mémoire collective multi-vocale, construite sur différentes échelles de temporalités réunies au sein du récit. Cette étude de la narration de l'attachement permet enfin de dégager quelques caractéristiques du lien partisan dans le Liban contemporain.

\section{Mots-clés}

Narration, mémoire collective, identité, Liban.

\begin{abstract}
Narration, because it implies both a dramatic presentation and an allocation of signification, stands as one of the main modes of construction and of representation of social ties. Focusing on the study of a Lebanese political party, the Free Patriotic Movement (FPM), this paper first mobilizes the concept of narrative identity to describe how a social group is entrenched into a specific storyline built by a particular emplotment. It is then demonstrated how this emplotment relies on a multivocal collective memory, constructed from various scales of temporality reunited in the story. Finally, the study of the narrative construction of affliation enables to highlight some of the characteristics of the political ties existing in contemporary Lebanon.
\end{abstract}

\section{Keywords}

Narrative, collective memory, identity, Lebanon.

\section{BIBLIOGRAPHIE}

Bakhtin M., The dialogic imagination: Four essays, Austin, University of Texas Press, 1981 [1930]. —-, Speech genres and other late essays, Austin, University of Texas Press, 1986.

Berger P. \& Luckmann T., The social construction of reality, Harmondsworth, Penguin Books, 1966.

Bourre J.-P., Génération Aoun. Vivre libre au Liban, Paris, Robert Laffont, 1990.

Brubaker R., "Au-delà de l'identité ", Actes de la recherche en sciences sociales, vol. 139, septembre 2001, p. 66-85.

Davie M., «Aoun ou le refus chrétien. Liban : octobre 1989-janvier 1990 », Temps Modernes, p. 536-537, 1990, p. 147-180.

Farrugia F., "Le syndrome narratif : théorie et terrain ", Cahiers internationaux de sociologie, $\mathrm{n}^{\circ} 127,2,2009$, p. 269-289.

Genette G., Figures I, Paris, Seuil, 1966.

—, Figures II, Paris, Seuil, 1969.

Halbwachs M., La mémoire collective, Paris, Seuil, 1997 [1950].

Harré R., "Positioning theory ", 2004, en ligne : www.massey.ac.nz/-alock/virtual/ positioning.doc.

Harré R. \& Van Langenhove L., Positioning Theory: Moral context of intentional action, Oxford, Blackwell, 1999.

Haugbolle S., "Public and private memory of the Lebanese civil war ", Comparative Studies of South Asia, Africa and the Middle-East, Duke University Press, vol. 25, n 1, 2005, p. 191-203. 
Jewsiewicki B., "Mémoire et représentation pour un vivre ensemble ", Espaces-Temps, $n^{\circ} 84-85-86,2004$, p. 187-192.

Lavabre M.-C., Le fil rouge. Sociologie de la mémoire communiste, Paris, Presses de la FNSP, 1994.

Lenclud G., "S'attacher. Le régime traditionnel de la protection en Corse », Terrain [en ligne], 21, 1993, p. $12:$ http://terrain.revues.org/3074.

Lombardo S., Liban Libre, Marseille, Éditions Transbordeurs, 2005.

Mermier F. \& Mervin S. (dir.), Leaders et partisans au Liban, Paris-Beyrouth, Karthala-IFPOIISMM, 2012.

Picard E., «Les dynamiques politiques des chrétiens au Liban. Changement de statut et crise du leadership ", Monde arabe Maghreb-Machrek, n 153, 1996, p. 3-21.

Qassem N., Hizbullah. The story from within, Londres, Saqi, 2005.

Ricour P., Du texte à l'action, Essais d'herméneutique II, Paris, Seuil, 1986.

Rivoal I., "Division, équilibre, médiation. Une lecture de l'opposition factionnelle dans le monde arabe ", Ateliers d'anthropologie, n 27, 2004, en ligne : http://ateliers.revues. org/8649?lang=en.

Rondeau D., Chroniques du Liban rebelle, 1988-1989, Paris, Grasset, 1991.

Salibi K., Une maison aux nombreuses demeures. L'identité libanaise dans le creuset de l'histoire, Paris, Naufal, 1989.

Sartre J.-P., La nausée, Paris, Folio, 1972.

Shuman A., "Entitlement and empathy in personal narrative ", in Bamberg M. (dir.), Narrative State of the art, op. cit., p. 175-184.

Somers M., "The Narrative Constitution of Identity: A Relational and Network Approach », Theory and Society, vol. 23, n 5, octobre 1994, p. 605-649.

Tilly C., From mobilization to revolution, Reading, Addison-Wesley, 1978.

Verret M., " Mémoire ouvrière, mémoire communiste ", Revue Française de Science Politique, vol. $34, n^{\circ} 3,1984$, p. 413-427. 